\title{
Transvaginal endoscopy and small ovarian endometriomas: unravelling the missing link?
}

\author{
S. Gordts • P. Puttemans • Sy. Gordts • M. Valkenburg • \\ I. Brosens $\cdot$ R. Campo
}

Received: 14 August 2013 / Accepted: 4 October 2013/Published online: 17 October 2013

(C) The Author(s) 2013. This article is published with open access at Springerlink.com

\begin{abstract}
The incidence of endometriosis in the infertile female is estimated to be between 20 and $50 \%$. Although the causal relationship between endometriosis and infertility has not been proven, it is generally accepted that the disease impairs reproductive outcome. Indirect imaging techniques and transvaginal laparoscopy now offer the possibility of an early stage diagnosis. Although it remains debated whether the disease is progressive, treatment in an early stage is recommendable as it carries less risk for ovarian damage, hence premature ovarian failure. Under water, inspection with the technique of transvaginal hydrolaparoscopy (THL) accurately shows the invagination of the ovarian cortex as minimal superficial lesions but with the presence of well-differentiated endometrial like tissue at the base, the lateral walls and especially the inner edges of the small endometrioma. An inflammatory environment is responsible for the formation of connecting adhesions with the broad ligament and lateral wall with invasion of endometrial-like tissue and formation of adenomyotic lesions. In around $50 \%$ of the small endometriomas, adhesiolysis is necessary at the site of invagination with opening of the cyst, to free the chocolate content and hereby recognize the underlying endometrioma. The detailed inspection of these early-stage endometriotic lesions at THL reunites the hypothesis of Sampson with the observation of Hughesdon.
\end{abstract}

Keywords Endoscopy · Transvaginal hydrolaparoscopy · Ovarian endometriosis $\cdot$ Endometrioma $\cdot$ Hydroflotation . Pathogenesis $\cdot$ Surgery

S. Gordts $(\bowtie) \cdot$ P. Puttemans $\cdot$ S. Gordts $\cdot$ M. Valkenburg $\cdot$

I. Brosens $\cdot$ R. Campo

Leuven Institute for Fertility and Embryology (L.I.F.E.),

Tiensevest 168, 3000 Leuven, Belgium

e-mail: Stephan.gordts@lifeleuven.be

\section{Introduction}

With an estimated incidence of 2-22\% in the general population, the incidence of endometriosis, diagnosed at laparoscopy in the infertile female, is reported to range between 20 and $50 \%$ [1]. Based on epidemiological data, it is generally accepted that endometriotic implants are related to an impaired reproductive outcome, although the causal relationship between endometriosis (i.e. the implants without adhesions) and infertility has not clearly been proven so far. Treatment of these lesions remains debatable as long as there is no proven causal relationship.

Several hypotheses have been formulated regarding the pathogenesis of endometriosis, the hypothesis of menstrual regurgitation and subsequent angiogenesis and implantation being the most widely accepted [2]. Regurgitated endometrial cells may implant on the ovarian surface, causing local bleeding and adhesions. Hughesdon [3] investigated a series of ovaries with the endometrioma in situ and demonstrated the invaginated cortex with fibrosis and adhesion formation. In contrast with other benign ovarian cysts, the ovarian endometrioma is formed as a pseudocyst. In situ ovarian cystoscopy and selected biopsies by Brosens et al. [4] confirmed these findings. As such the basis and wall of this cyst is formed by inverted ovarian cortex, harbouring primordial and primary follicles. Strictly speaking, this also means the ovarian endometrioma is in fact an extra-ovarian pathology. However, with the aging of the endometrioma, the invaginated cortex gradually thickens by smooth muscle metaplasia and fibrosis and its appearance at cystoscopy changes from pearlwhite to yellow-white and finally black and fibrotic [5].

Other theories are the formation of endometriomas by metaplasia of invaginated coelomic epithelium [6] questioning the necessity of the presence of adhesions with the broad ligament as suggested by Hughesdon [3]. In a recent paper, Vercellini et al. [7] conclude that bleeding from a corpus 
luteum appears to be a critical event in the development of endometriomas and suggest that a possible alternative source of entrapped blood is a cystic corpus luteum developing along an ovarian cortex adherent to the pelvic sidewall. The patients studied in this paper all have been operated previously for endometriosis and were then followed by ultrasound for a period of 2 years. However, Ferrari et al. [8] recently described an ovarian endometriotic cyst in a 26-year-old girl with prepubertal hypopituitarism who never ovulated in her life and was treated with hormone replacement therapy.

Follicular growth can start from the basis of the invaginated ovarian cortex and co-exist with the endometriotic cyst. Communicating luteal cysts have been observed by Sampson [9] in $9 \%$ of his cases.

\section{Diagnosis}

Transvaginal sonography (TvS) is widely considered as a useful method for early detection of the ovarian endometrioma and seems a reliable technique to exclude significant ovarian endometriosis in infertile patients. However, a recent critical review on the accuracy of ultrasound in the diagnosis of endometriosis found that the available prospective studies all included endometriomas with a diameter of at least $14 \mathrm{~mm}$ [10]. So its accuracy in the diagnosis of endometriomas of less than $14 \mathrm{~mm}$ is not known. In our consecutive series of 169 patients where endometriosis was diagnosed at transvaginal hydrolaparoscopy (THL), preoperative TVU only detected $45 \%$ out of 11 endometriomas smaller than $15 \mathrm{~mm}$ (unpublished data).

At laparoscopy, subtle lesions and superficial adhesions are frequently missed as they are masked due to the high abdominal pressure of the $\mathrm{CO}_{2}$ pneumoperitoneum. The use of hydroflotation [11] prevents the collapse of filmy adhesions and allows the visualization of both subtle lesions and their neovascularization.

\section{Transvaginal hydrolaparoscopy}

THL has been described as a more sensitive technique than standard laparoscopy to detect subtle ovarian lesions [12]. The advantages of transvaginal endoscopy are numerous.

First, as distension medium, a pre-warmed Ringer lactate or Hartmann solution is used keeping the organs afloat and enabling visualization of early endometriotic lesions of the peritoneum and ovary such as free-floating adhesions and neoangiogenesis [12]. Secondly, as the visual axis is along the longitudinal tubo-ovarian axis, no extra manipulation is needed for a close inspection of the ovarian fossa avoiding disruption of the adhesions and rupture of the endometrioma by manipulating the ovary [13]. Thirdly, the watery distension medium enables a clear and highly contrasting visualization of the different planes of cleavage with their vascularisation, allowing a careful dissection and coagulation. Lastly, using 5 Fr instruments and the procedure being performed under water, there is a diminished risk of postoperative adhesion formation. All the procedures are performed in an outpatient hospital setting with patients returning home the same day. In the absence of a panoramic view, there is no place for major operative procedures.

The operative procedure for endometriomata at the time of THL can be performed as described by Gordts et al. [14]. If not detected at ultrasound, the diameter of the endometriotic cyst is estimated during the surgical procedure by comparison to the diameter of the biopsy forceps. Targeted biopsies of endometriomas during hydroflotation are obtained as previously described [14].

The characteristic features, which are identified by the hydroflotation technique, include the retraction and invagination of the cortex, microvascularisation, pigmentation, and free-floating adhesions and in some cases, adhesions with the fossa ovarica. After adhesiolysis and opening of the cyst at the site of invagination by micro scissors and/or a bipolar needle, the wall stays open, typically showing the same pearl-white appearance as the outer cortex, lined by a highly vascularised, polypoidal tissue [15].Targeted biopsies can confirm superficial endometriotic tissue, which usually are found at the edges and cortical tissue at the base of the cyst.

The early stages of invagination of the ovarian cortex can accurately been observed in cases of small endometriotic spots or endometriomata of 5-10 $\mathrm{mm}$. What looks like an endometriotic vesicle implanted at the ovarian surface is in fact hiding an underlying invagination of the ovarian cortex covered by tiny adhesions retaining the typical brownish fluid (Fig. 1a). After the removal of the superficial adhesions and drainage of the fluid, active endometrial-like tissue and neoangiogenesis can be seen at the inside of the extraovarian pseudocyst. This also indicates that the presence of adhesions with the peritoneum of the lateral pelvic wall is not required to result in the development of a small invagination of the cortex, the ceiling of which consists of tiny adhesions. The subsequent shedding of the endometriotic implants at the inside results in the formation of the typical brownish content that is trapped inside the small endometrioma. In the more severe stages of the disease, adhesions with the pelvic sidewall can be formed (Fig. 1b). These adhesions are always connecting the site of invagination with the peritoneum of the pelvic wall (Fig. 2a). Although dealing with small lesions, by careful dissection, there is not only an invagination of the ovarian cortex with the typical lesions insight but also the peritoneal wall opposing the invagination is invaginated itself, and invaded by endometrium like tissue (Fig. 2). There seems to be a continuum between the ovarian endometrioma and the 


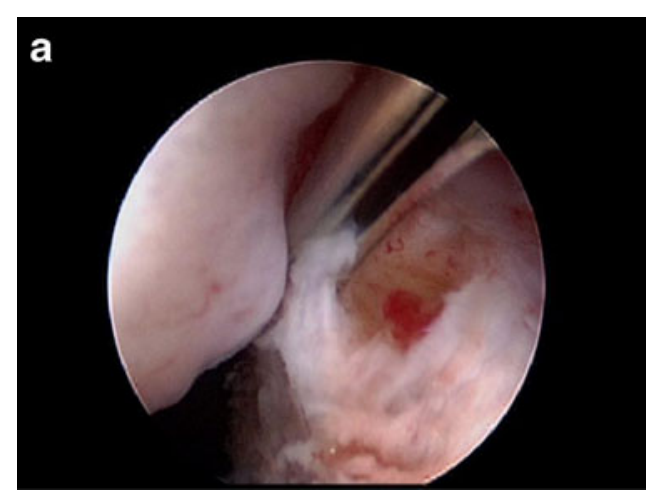

Fig. 1 A A brownish vesicle upon the ovarian surface: after removal of the superficial adhesions and leakage of the brownish fluid small endometrial-like tissue can clearly be seen at the base of this beginning

peritoneal lesions covered by adhesions. Once the adhesions are removed, the peritoneal wall lesion can be opened, showing the same pathology as the inside of the ovarian

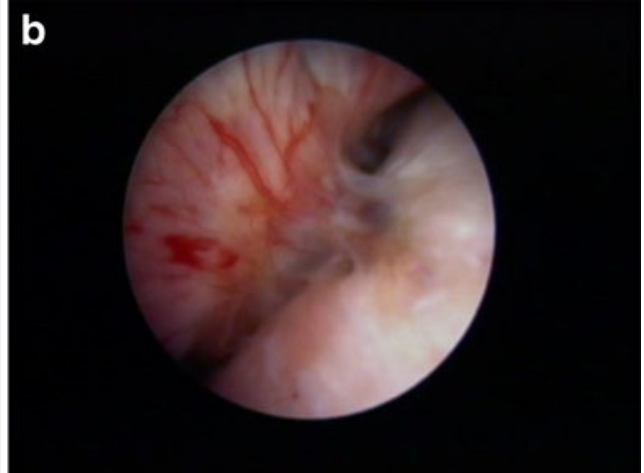

invaginating cortex. B Beginning of adhesive process between small ovarian endometriotic lesion and pelvic wall. Remark the neoangiogenesis upon the peritoneal surface
Fig. 2 A Fixed adhesion between ovary and pelvic wall at the site of invagination; $\mathbf{B}$ freed ovary, clearly showing the invagination of the cortex; $\mathbf{C}$ opening of endometrioma of $1.5 \mathrm{~cm}$ with bipolar needle at site invagination; $\mathbf{D}$ insight view clearly showing the presence of endometrial-like tissue; $\mathbf{E}$ ablative surgery using bipolar probe; $\mathbf{F}$ after ablation with bipolar: mark the white color of the base of the cyst like the normal ovarian cortex. No presence of carbonization using the bipolar under water. The image shows clearly the invagination of the cortex
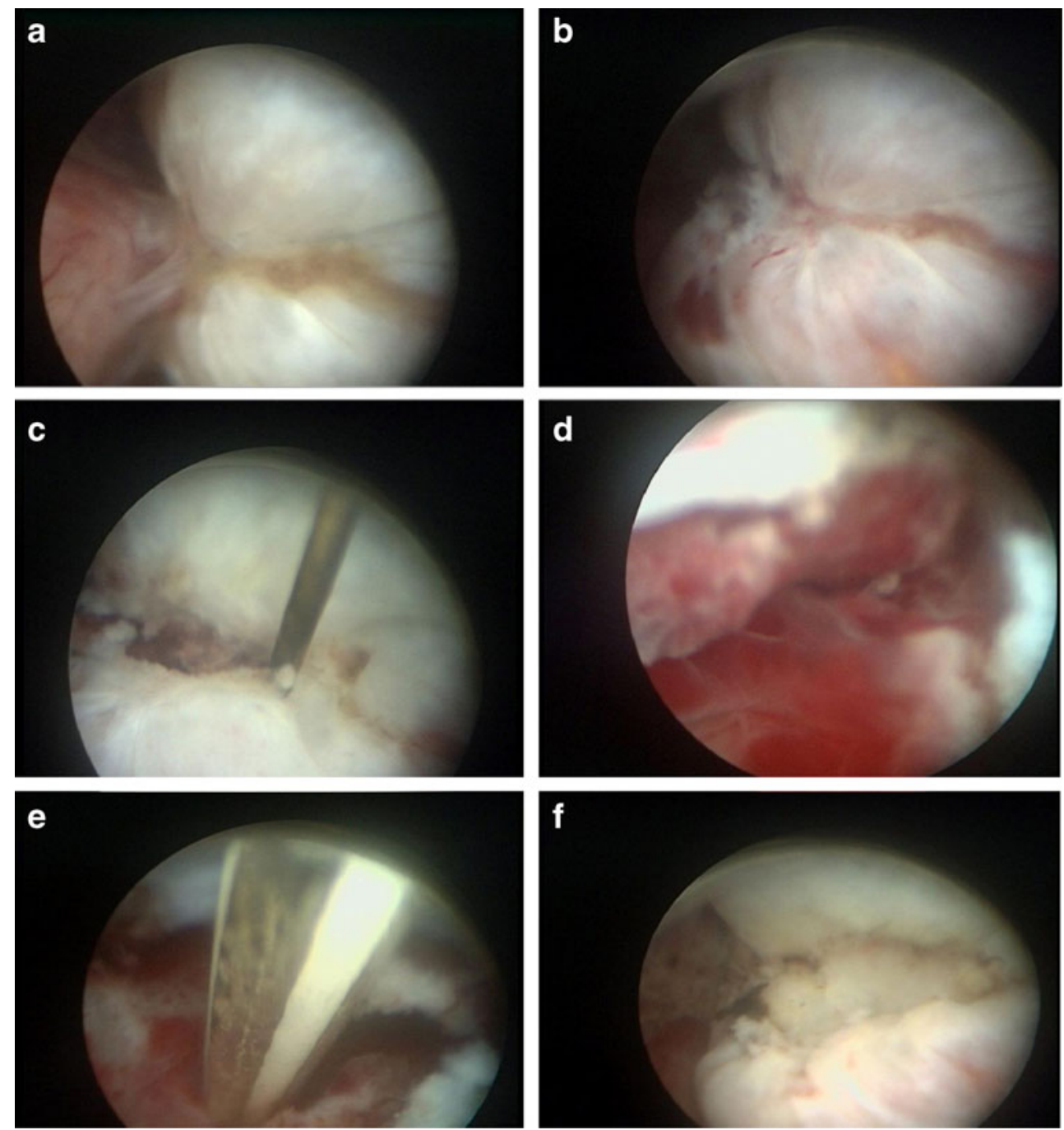


\section{Discussion}

THL is an appropriate technique for the detection and surgery of small ovarian endometriomas. Transvaginal endoscopy allows the accurate exploration of the initial stages of ovarian and peritoneal endometriosis. Small endometrioma of 5-10 $\mathrm{mm}$ can be formed on the ovarian surface in the absence of connecting adhesions with the pelvic wall. What initially presents as a small superficial lesion without significance, appearing like a small cicatricial line surrounding a brownish spot, can surprisingly hide a much deeper infiltration in the ovarian cortex with the typical inflammatory neovascularization and red endometrial like implants. In approximately half of the cases, active adhesiolysis with micro scissors is needed to discover these deeper invaginations. In the case of connecting adhesions with the pelvic wall, the invagination is more pronounced, with a stronger appearance of the inflammatory reaction and the neoangiogenesis.

Our observational study clearly showed that in cases of small endometriomas the wall is formed by invaginated ovarian cortex, sealed off by fusion and/or adhesions of superficial endometriotic implants at the surface, resulting in an extraovarian pseudocyst. As invagination can clearly be visualized in small ovarian endometrioma without connecting adhesions towards the peritoneum of the lateral pelvic wall, these invaginations and their chocolate content are not due to the fixed adhesion formation preventing endometriotic deposits from escaping, as suggested by Jones et al. [16]. Bleeding originates from the active endometriumal-like tissue covering the inside and mostly the inner edges of the pseudocyst, in the presence of active neoangiogenesis, offering a double target for visually directed endoscopic therapy. The inflammatory reaction originating from the endometrial implants on the ovarian surface initiates the process of small yet strong surface adhesions causing a closure of the invading endometrial tissue with invagination of the cortex. Further inflammatory reaction causes adhesions with the broad ligament originating at the site of invagination and enabling invasion of endometriotic tissue both into the ovary and into the broad ligament.

In none of the operated cases of small ovarian endometriomas, we noticed the presence of a follicular cyst or a connecting corpus luteum, the inside of which has a completely different endoscopic appearance with thick layers of vivid orange-red lutein cells. The hypothesis of Vercellini et al. [7] stating that ovarian endometrioma originates from entrapped tissue in the developing and ovulating follicle cannot be confirmed by our findings.

There is no doubt that from the small brown vesicle on the ovarian surface up to the small endometrioma with or without adhesions towards the pelvic wall, invagination of the ovarian cortex can clearly be demonstrated in each and every case. In contrast with standard laparoscopy, THL allows very detailed inspections of the ovarian endometriotic lesions and as such provides the missing link in the pathogenesis of the ovarian endometrioma, reuniting the hypothesis of Sampson with the observations of Hughesdon.

Small endometriomas can be treated surgically during a THL procedure [15]. The present experience confirms our previous report that THL provides an atraumatic single access approach for the surgical reconstruction of small ovarian endometriomas. During THL, the whole surface of the floating ovary can be inspected in detail, with special emphasis to its posterior side and the "roof" of the ovarian fossa, without any risk that adhesions are ruptured, start bleeding and collapse before they are inspected. Endometriotic adhesions may contain endometriotic cells and require to be visualized and ablated. The classical steps of ovarian reconstruction are achieved without complex instrumental manipulation by complete adhesiolysis and mobilization of the ovary, followed by opening of the pseudocavity at the site of invagination and rinsing of the chocolate content and finally bipolar ablation of the superficial endometriotic tissue. As the entire procedure is performed under hydro flotation of the ovary, bipolar coagulation is used, minimizing the risk of postoperative adhesion formation.

Indirect imaging techniques and THL now offer the possibility of an early diagnosis of small ovarian endometriotic cysts. The clinical significance of the detection and surgical treatment of small endometriomas in patients with infertility requires further investigation. However, it can be speculated that ablation of an ovarian endometrioma, even small or medium in size, in patients with infertility is as beneficial, if not more, as ablation of minimal or mild endometriosis. The size of the endometrioma is not the only factor determining the severity of the disease. As disease and diameter progresses, the negative impact of fibrosis and smooth muscle metaplasia upon the ovarian reserve are becoming more important [17]. Although today there is growing concern of impaired ovarian function after ovarian endometrioma surgery, surgery in an early stage has the advantage of being less traumatic, and the operative procedure can be performed before the onset of intra ovarian fibrosis and smooth muscle metaplasia [18]. Operating in an early stage carries the potential advantage of a lower risk of recurrence. There is increasing evidence that the diagnosis of endometriosis, whatever its stage, is important in patients with infertility. In a retrospective study of patients with unexplained infertility Akande et al. [19] found that when these patients had minimal or mild endometriosis, which was left untreated, the time to natural conception was significantly prolonged in comparison with patients without lesions. This together with the results of the Canadian Collaborative Group study on ablation of minimal and mild endometriosis [20] supports the view that detecting and treating these lesions as early as possible is beneficial in subfertile women. An Italian study could neither reject nor confirm this observation [21]. A recent review combining the 
results of both trials into a meta-analysis showed that surgical treatment is more favourable than expectant management (odds ratio for pregnancy 1.7; $95 \%$ confidence interval 1.1-2.5) [22]. As there are at present no specific markers available indicating the aggressiveness of the lesions and the risk of progressivity, treating in this early stage is recommended. More research is needed to evaluate the real risk of progressivity of these lesions.

In conclusion, the visual diagnosis of small ovarian endometrioma at THL is reliable and accurate. It enables to analyse the process of the ovarian endometriosis from his early onset up till the formation of a small endometrioma. The obtained data confirm the extraovarian localization of the endometrioma with the secondary formation of adhesions.

Conflict of interest Stephan Gordts, Consultant Karl Storz, Germany, declares that his relation with the company has no impact upon the scientific value and content of the submitted document.

Open Access This article is distributed under the terms of the Creative Commons Attribution License which permits any use, distribution, and reproduction in any medium, provided the original author(s) and the source are credited.

\section{References}

1. Moen MH (1987) Endometriosis in women at interval sterilization. Acta Obstet Gynecol Scand 66(5):451-454

2. Sampson JA (1927) Peritoneal endometriosis due to the menstrual dissemination of endometrial tissue into the peritoneal cavity. Am J Obstet Gynecol 14:422-469

3. Hughesdon PE (1957) The structure of endometrial cysts of the ovary. J Obstet Gynaecol Br Emp 44:481-487

4. Brosens IA, Puttemans PJ, Deprest J (1994) The endoscopic localization of endometrial implants in the ovarian chocolate cyst. Fertil Steril 61:1034-1038

5. Darwish AM, Amin AF, El-Feky MA (2000) Ovarioscopy, a technique to determine the nature of cystic ovarian tumors. J Am Assoc Gynecol Laparosc 7:539-544

6. Nisolle M, Donnez J (1997) Peritoneal endometriosis, ovarian endometriosis, and adenomyotic nodules of the rectovaginal septum are three different entities. Fertil Steril 68:585-596
7. Vercellini P, Somigliana E, Vigano P, Abbiati A, Barbara G, Fedele L (2009) 'Blood On The Tracks' from corpora lutea to endometriomas. BJOG 116:366-371

8. Ferrari S, Persico P, Di Puppo F, Garavaglia E, Viganò P, Candiani M (2012) An ovarian endometriotic cyst in a patient with prepubertal hypopituitarism due to a craniopharyngioma: a clue for endometrioma pathogenesis. Eur J Obstet Gynecol Reprod Biol 164(1):115-116

9. Sampson JA (1921) Perforating hemorrhagic (chocolate) cysts of the ovary. Arch Surg 3:245-323

10. Moore J, Copley S, Morris J, Lindsell D, Golding S, Kennedy S (2002) A systematic review of the accuracy of ultrasound in the diagnosis of endometriosis. Ultrasound Obstet Gynecol 20:630-634

11. Laufer MR, Goitein L, Bush M, Cramer DW, Emans SJ (1997) Prevalence of endometriosis in adolescent girls with chronic pelvic pain not responding to conventional therapy. J Pediatr Adolesc Gynecol 10:199-202

12. Brosens I, Gordts S, Campo R (2001) Transvaginal hydrolaparoscopy but not standard laparoscopy reveals subtle endometriotic adhesions of the ovary. Fertil Steril 75:1009-1012

13. Gordts S, Campo R, Rombauts L, Brosens I (1998) Transvaginal hydrolaparoscopy as an outpatient procedure for infertility investigation. Hum Reprod 13:99-103

14. Gordts S, Campo R, Brosens I, Puttemans P (2003) Endometriosis: modern surgical management to improve fertility. Baillieres Best Pract Res Clin Obstet Gynaecol 17:275-287

15. Gordts S, Campo R, Brosens I (2002) Experience with transvaginal hydrolaparoscopy for reconstructive tubo-ovarian surgery. Reprod BioMed Online 4(Suppl 3):72-75, Review

16. Jones KD, Fan A, Sutton CJ (2002) The ovarian endometrioma: why it is so poorly managed? Indicators from an anonymous survey. Hum Reprod 17:845-849

17. Fukunaga M (2000) Smooth muscle metaplasia in ovarian endometriosis. Histopathology 36:348-352

18. Brosens I, Gordts S., Puttemans P, Benagiano P (2013) Pathophysiology of the ovarian endometrioma as a basis for management. RBM online submitted

19. Akande VA, Hunt LP, Cahill DJ, Jenkins JM (2004) Differences in time to natural conception between women with unexplained infertility and infertile women with minor endometriosis. Hum Reprod 19: 96-103

20. Marcoux S, Maheux R, Bérubé S, and the Canadian Collobarative Group on Endometriosis (1997) Laparoscopic surgery in infertile women with minimal or mild endometriosis. N Engl J Med 337: 217-222

21. Parazzini F (1999) Ablation of lesions or no treatment in minimalmild endometriosis in infertile women: a randomized trial. Hum Reprod 14:1332-1334

22. Olive DL, Pritts EA (2002) The treatment of endometriosis: a review of the evidence. Ann N Y Acad Sci 955:360-372 